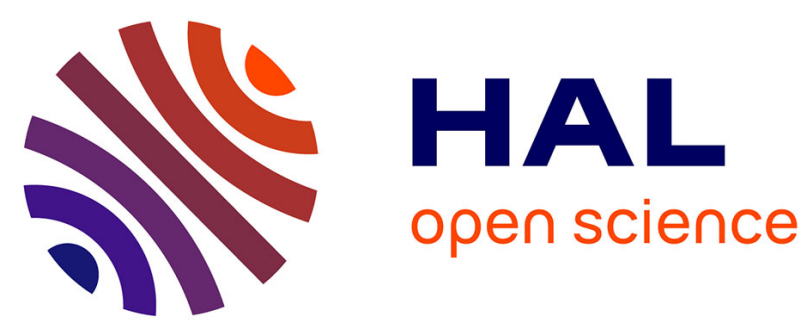

\title{
Étude du transfert d'excitation par émission spontanée II. Vérifications expérimentales dans le cas d'une irradiation laser application au niveau 2 p2 du néon Martial Ducloy, Elisabeth Giacobino-Fournier, Bernard Decomps
}

\section{- To cite this version:}

Martial Ducloy, Elisabeth Giacobino-Fournier, Bernard Decomps. Étude du transfert d'excitation par émission spontanée II. Vérifications expérimentales dans le cas d'une irradiation laser application au niveau 2 p2 du néon. Journal de Physique, 1970, 31 (7), pp.533-543. 10.1051/jphys:01970003107053300 . jpa-00206936

\section{HAL Id: jpa-00206936 https://hal.science/jpa-00206936}

Submitted on 1 Jan 1970

HAL is a multi-disciplinary open access archive for the deposit and dissemination of scientific research documents, whether they are published or not. The documents may come from teaching and research institutions in France or abroad, or from public or private research centers.
L'archive ouverte pluridisciplinaire HAL, est destinée au dépôt et à la diffusion de documents scientifiques de niveau recherche, publiés ou non, émanant des établissements d'enseignement et de recherche français ou étrangers, des laboratoires publics ou privés. 


\title{
ÉTUDE DU TRANSFERT D'EXCITATION PAR ÉMISSION SPONTANÉE \\ II. VÉRIFICATIONS EXPÉRIMENTALES DANS LE CAS D'UNE IRRADIATION LASER APPLICATION AU NIVEAU $2 p_{2}$ DU NÉON $\left(^{1}\right)$
}

\author{
par Martial DUCLOY, Elisabeth GIACOBINO-FOURNIER et Bernard DECOMPS \\ Faculté des Sciences de Paris, Laboratoire de Spectroscopie Hertzienne de l'E. N. S., \\ associé au C. N. R. S. 24, rue Lhomond, Paris, Ve
}

(Reçu le 12 janvier 1970)

\begin{abstract}
Résumé. - La lumière de fluorescence émise par des atomes de néon soumis à un champ magnétique et placés à l'intérieur de la cavité d'un laser à gaz est modifiée par l'irradiation laser. Dans le cas de l'irradiation par la raie $\lambda=1,52 \mu\left(2 s_{2}-2 p_{1}\right)$ du néon, les modifications observées sur les raies de fluorescence issues des niveaux $2 s_{2}$ et $2 p_{4}$ permettent de vérifier la théorie de transfert d'excitation par émission spontanée $\left(2 s_{2} \rightarrow 2 p_{4}\right)$, telle qu'elle a été présentée dans l'article I ( ${ }^{2}$ ). La méthode mise au point à l'aide de ces vérifications permet alors d'étudier la relaxation de la population et de l'alignement du niveau $2 p_{2}$ également peuplé par émission spontanée à partir du niveau $2 s_{2}$. On a pu mesurer les sections efficaces correspondantes et la durée de vie naturelle de ce niveau. On a aussi mis en évidence un phénomène de réabsorption des raies de fluorescence issues du niveau $2 p_{2}$ par les états métastables $1 s$ du néon.

L'irradiation laser à la longueur d'onde $\lambda=6401 \AA\left(3 s_{2}-2 p_{2}\right)$ permet de retrouver les résultats précédents. Dans ce cas, on a pu observer la création d'alignement dans le niveau $2 p_{2}$ simultanément par le laser et par l'émission spontanée sur la raie laser elle-même. Cette dernière étude a mené à la détermination de la probabilité de transition de la raie $\lambda=6401 \AA$ :
\end{abstract}

$$
\gamma_{b a}=1,2 \pm 0,5 \times 10^{6} \mathrm{~s}^{-1} \text {. }
$$

Abstract. - The fluorescence light emitted by excited neon atoms placed inside a laser cavity is strongly changed when they are illuminated by a $1,52 \mu$ or $6401 \AA$ laser beam, in the presence of a magnetic field.

In the case of $1,52 \mu\left(2 s_{2}-2 p_{1}\right)$ laser irradiation, a detailed study of this effect on the fluorescence lines emitted by the $2 s_{2}$ and $2 p_{4}$ levels $\left(2 p_{4}\right.$ is populated by spontaneous emission from $2 s_{2}$ ) shows good agreement with a previously published theory of excitation transfer by spontaneous emission (2). The relaxation of the $2 p_{2}$ level has been studied by the same method. Collision cross sections for population and alignment, and natural width have been measured. Multiple scattering phenomena on $2 p_{2}-1 s$ lines have been observed.

Identical results are obtained for $6401 \AA\left(3 s_{2}-2 p_{2}\right)$ laser excitation. In this case, alignment of the $2 p_{2}$ level is produced both by the laser beam and by spontaneous emission on the laser line itself. The transition probability, for the $6401 \AA$ line has been measured :

$$
\gamma_{b a}=(1.2 \pm 0.5) \times 10^{6} \mathrm{~s}^{-1} .
$$

A. Introduction. - Nous nous proposons de vérifier les prévisions de la théorie du transfert d'excitation, telle qu'elle a été présentée dans l'article $I\left({ }^{2}\right)$ et de l'appliquer à la mesure de temps de relaxation des niveaux atomiques. Pour cela, nous réalisons une expérience de pompage optique en irradiant avec un

(1) Cette étude a été réalisée avec l'aide de la D. R. M. E.

(2) Toutes les références, formules et paragraphes de cet article théorique sont précédés de I. Ducloy M. et Dumont M., J. Physique, 1970, 31, 419. laser hélium-néon une cellule contenant un mélange d'hélium et de néon excité par une décharge. La décharge peuple de façon isotrope tous les niveaux du néon tandis que le pompage optique introduit une perturbation anisotrope sélectivement dans les niveaux supérieur et inférieur correspondant à la transition laser utilisée. Ces niveaux se désexcitent par cascades radiatives vers des niveaux d'énergie inférieure. Les atomes sont placés dans un champ magnétique uniforme orienté suivant la direction de propagation du faisceau laser. Nous observons les cascades de désex- 
citation grâce à l'étude des variations en fonction du champ magnétique de l'intensité et de la polarisation de la lumière de fluorescence émise par les divers niveaux de la cascade.

Les vérifications expérimentales ont porté sur la forme de l'effet Hanle de transfert du niveau $2 p_{4}$ et sur le taux de transfert par la transition $2 s_{2}-2 p_{4}$, dans le cas d'une excitation laser résonnante pour la transition $2 s_{2}-2 p_{1}(\lambda=1,52 \mu)$. La méthode mise au point sur le niveau $2 p_{4}$ a permis alors l'étude du niveau $2 p_{2}$ également peuplé par transfert à partir du niveau .supérieur $2 s_{2}$ de la raie laser $\lambda=1,52 \mu$ (Fig. 1).

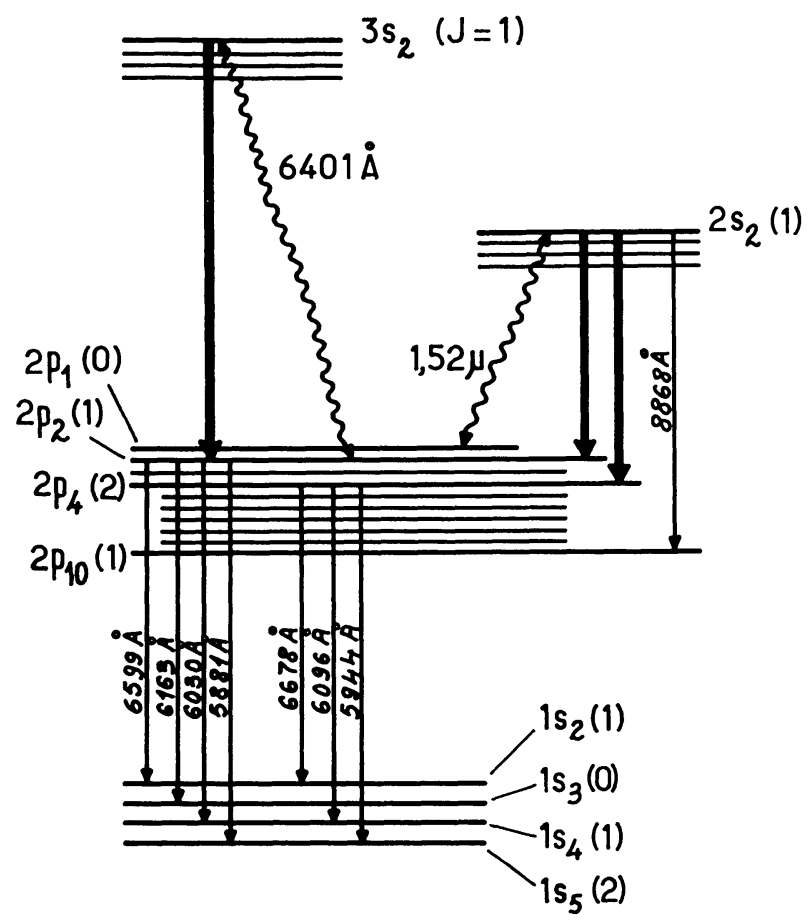

FIG. 1. - Niveaux excités du néon (notation de Paschen).

Les résultats obtenus ont été finalement confrontés avec ceux que l'on obtient à l'aide d'une excitation laser sur la transition $3 s_{2}-2 p_{2}(\lambda=6401 \AA)$. Dans ce dernier cas, les deux effets, excitation directe et excitation par transfert, sont présents.

1. Le MONTAGE EXPÉRIMENTAL (Fig. 2). - Le montage expérimental employé pour obtenir l'excitation

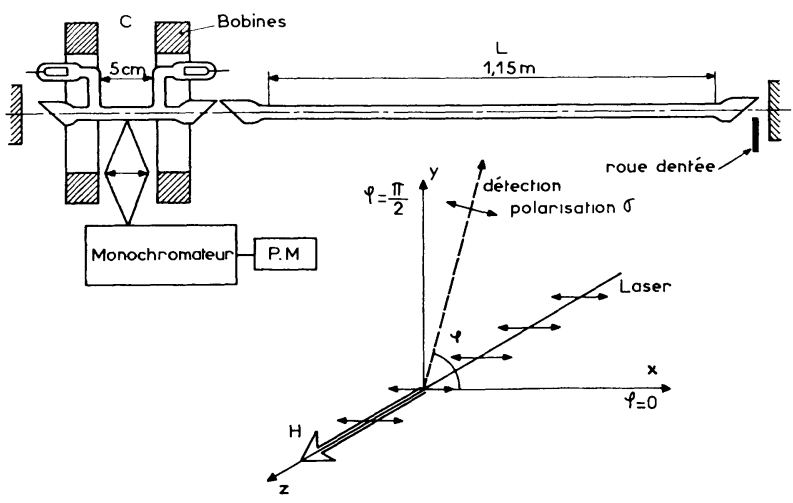

FIG. 2. - Montage expérimental et directions d'observation de la lumière de fluorescence. par une raie laser est celui déjà décrit dans des publications antérieures [1] [2] [3]. Entre deux miroirs, nous disposons une cellule $\mathrm{C}$ et un tube amplificateur $\mathrm{L}$. L'oscillation laser est assurée par le tube L, beaucoup plus long que $\mathrm{C}$, dans lequel la nature du mélange hélium-néon et les conditions de décharge sont ajustées pour obtenir le maximum d'intensité lumineuse. Nous avons fait osciller le montage sur les longueurs d'onde $6401 \AA$ et $1,52 \mu$. Le choix de la longueur d'onde est assuré par les miroirs multicouches diélectriques dont le coefficient de réflexion n'est proche de un que pour une faible gamme de longueur d'onde. De plus, pour obtenir la raie $6401 \AA$ au détriment de la raie $6328 \AA$ très proche et plus intense, il faut choisir convenablement le rayon de courbure des miroirs du laser [20].

La cellule $C$ est remplie d'un mélange d'hélium et de néon en proportions variables, qui est excité par une décharge continue d'intensité réglable. Deux bobines en position de Helmholtz permettent d'appliquer un champ magnétique axial sur $\mathrm{C}$. Les fenêtres à incidence de Brewster, qui se trouvent aux extrémités de $\mathrm{C}$ et $\mathrm{L}$, imposent une polarisation rectiligne au faisceau laser ( $\sigma$ «cohérent » par rapport au champ magnétique).

L'étude des atomes de $\mathrm{C}$ se fait grâce à l'observation de la polarisation de la lumière de fluorescence. Nous étudions les raies de fluorescence émises par la cellule $\mathrm{C}$, perpendiculairement à l'axe du laser, dans des directions parallèle $(\varphi=0)$ et perpendiculaire $(\hat{\varphi}=\pi / 2)$ au plan de polarisation de l'onde laser (Fig. 2). Dans chacune des deux directions, un miroir semi-transparent permet de dédoubler le faisceau et d'observer simultanément deux raies de fluorescence.

Dans le cas de l'irradiation à $1,52 \mu$, il faut observer simultanément les raies de fluorescence du niveau $2 s_{2}$ qui sont dans le proche infra-rouge et les raies de fluorescence des niveaux $2 p_{n}$ qui sont dans le visible. Pour cela les miroirs semi-transparents sont remplacés par des miroirs «froids» (Athervex R. fabriqués par M. T. O.) qui laissent passer l'infra-rouge et réfléchissent le visible. Les polariseurs pour les raies visibles, transparents pour l'infra-rouge, sont placés près de la cellule. Les polariseurs infra-rouge, ne laissant pas passer la lumière visible, sont placés après les miroirs «froids ". Il a été vérifié que la partie du système optique se trouvant devant ces polariseurs ne détériore pas la polarisation de la lumière de fluorescence, dans les limites de précision des expériences. Sur chacune des quatre voies d'observation, la raie choisie est détectée par un photomultiplicateur placé derrière un monochromateur.

Les photomultiplicateurs 150 CVP (Radiotechnique) utilisés pour les raies infra-rouge présentent un courant d'obscurité très fort et, par suite, un bruit important. Pour diminuer ce courant d'obscurité, on les refroidit à environ $-30^{\circ} \mathrm{C}$ par un courant d'azote. Comme la sensibilité des photomultiplicateurs dépend de la température, celle-ci est stabilisée à quelques degrés près. 
2. LA LUMIÈRE DE FLUORESCENCE. - Rappelons quelques formules sur la lumière de fluorescence.

L'intensité de la polarisation $\sigma$ (polarisation perpendiculaire à $H$ ) de la raie de fluorescence $\beta \rightarrow \alpha$ est donnée par [4]

$$
\begin{aligned}
L_{\mathrm{F}, \beta \alpha}^{\sigma_{/ \prime}}= & I_{0} \times \\
& \times\left[\frac{{ }_{\beta} \rho_{0}^{0}(H)}{3 \sqrt{2 J_{\beta}+1}}+(-)^{J_{\beta}+J_{\alpha}+1}\left\{\begin{array}{ccc}
1 & 1 & 2 \\
J_{\beta} & J_{\beta} & J_{\alpha}
\end{array}\right\}\right. \\
& \left.\times\left(\frac{{ }_{\beta} \rho_{0}^{2}(H)}{\sqrt{6}}+\frac{{ }_{\beta} \rho_{2}^{2}(H)+{ }_{\beta} \rho_{-2}^{2}(H)}{2}\right)\right]
\end{aligned}
$$

pour la direction $\varphi=0$, et

$$
\begin{aligned}
L_{\mathrm{F}, \beta \alpha}^{\sigma_{\perp}}= & \\
=I_{0} & {\left[\frac{{ }_{\beta} \rho_{0}^{0}(H)}{3 \sqrt{2 J_{\beta}+1}}+(-)^{J_{\beta}+J_{\alpha}+1}\left\{\begin{array}{ccc}
1 & 1 & 2 \\
J_{\beta} & J_{\beta} & J_{\alpha}
\end{array}\right\} \times\right.} \\
& \left.\times\left(\frac{{ }_{\beta} \rho_{0}^{2}(H)}{\sqrt{6}}-\frac{{ }_{\beta} \rho_{2}^{2}(H)+{ }_{\beta} \rho_{-2}^{2}(H)}{2}\right)\right] \quad(\mathrm{II}, 2)
\end{aligned}
$$

pour la direction $\varphi=\pi / 2$ (Fig. 2).

Les notations employées sont celles qui ont été déjà utilisées dans l'article précédent (I).

L'opposition de ces deux signaux nous donne directement une grandeur proportionnelle à $\operatorname{Re}\left[{ }_{\beta} \rho_{2}^{2}(H)\right]$ : c'est l'effet Hanle, qui nous permet d'obtenir $\Gamma_{\beta}(2)$. L'observation simultanée de $L_{\sigma_{/ /}}(H)$ et $L_{\sigma_{\perp}}(H)$ nous permet de distinguer la partie "anisotrope » de la lumière de fluorescence (liée à l'alignement transversal ${ }_{\beta} \rho_{ \pm 2}^{2}$ ) de la partie « isotrope » (liée à la population ${ }_{\beta} \rho_{0}^{0}$ et à l'alignement longitudinal $\left.{ }_{\beta} \rho_{0}^{2}\right)\left({ }^{3}\right)$, et d'obtenir par suite le taux d'anisotropie en champ magnétique nul [4] :

$$
\begin{gathered}
R_{\beta \alpha}=\frac{L^{\sigma_{\perp}}(H=0)-L^{\sigma / l}(H=0)}{L^{\sigma} \perp(H=0)+L^{\sigma / /}(H=0)}= \\
=\frac{(-)^{J_{\beta}+J_{\alpha}}\left\{\begin{array}{ccc}
1 & 1 & 2 \\
J_{\beta} & J_{\beta} & J_{\alpha}
\end{array}\right\} \cdot \frac{{ }_{\beta} \rho_{2}^{2}(0)+{ }_{\beta} \rho_{-2}^{2}(0)}{2}}{3 \frac{{ }_{\beta} \rho_{0}^{0}(0)}{\sqrt{2 J_{\beta}+1}}+(-)^{J_{\beta}+J_{\alpha}+1}\left\{\begin{array}{ccc}
1 & 1 & 2 \\
J_{\beta} & J_{\beta} & J_{\alpha}
\end{array}\right\} \frac{{ }_{\beta} \rho_{0}^{2}(0)}{\sqrt{6}}} .
\end{gathered}
$$

Envisageons le cas d'une excitation laser de polarisation rectiligne en champ magnétique nul. Si on prend l'axe de quantification suivant la polarisation du laser, les symétries de l'excitation font que le laser, quelle

(3) En champ magnétique fort, l'alignement transversal est nul. $L^{\sigma} / /$ et $L^{\sigma}{ }_{\perp}$ sont donc théoriquement égaux. Cette remarque nous permet d'égaler la sensibilité des deux voies d'observation. Cette opération est nécessaire pour obtenir l'effet Hanle, par opposition des deux voies, ou pour mesurer le taux d'anisotropie $R_{\beta \alpha}$. que soit son intensité, ne peut créer que des grandeurs longitudinales ${ }_{\beta} \rho_{0}^{k}$ [5]. Par une rotation de $\pi / 2$, on peut amener la polarisation du laser sur $O x$. Les formules de rotation des tenseurs irréductibles nous donnent, pour les composantes de l'alignement, les relations [5]:

$$
{ }_{\beta} \rho_{2}^{2}(0)={ }_{\beta} \rho_{-2}^{2}(0)=-{ }_{\beta} \rho_{0}^{2}(0) \sqrt{\frac{3}{2}} .
$$

Ces relations sont valables aussi bien pour les niveaux de la transition laser que pour les niveaux atteints par cascade radiative. Elles permettent d'écrire $R_{\beta \alpha}$ sous la forme simple

$$
=\frac{(-)^{J_{\beta}+J_{\alpha}+1} 3 \cdot\left\{\begin{array}{ccc}
1 & 1 & 2 \\
J_{\beta} & J_{\beta} & J_{\alpha}
\end{array}\right\}}{\sqrt{\frac{2}{3\left(2 J_{\beta}+1\right)} \cdot \frac{{ }_{\beta} \rho_{0}^{0}(0)}{{ }_{\beta}^{2} \rho_{0}^{2}(0)}+(-)^{J_{\beta}+J_{\alpha}+1}\left\{\begin{array}{ccc}
1 & 1 & 2 \\
J_{\beta} & J_{\beta} & J_{\alpha}
\end{array}\right\} .} .}
$$

Présentons maintenant quelques résultats théoriques valables dans le cas d'une irradiation laser résonnante pour une transition entre niveaux de moments cinétiques $J=1 \leftrightarrow J=0, J=1 \leftrightarrow J=1$ ou $J=\frac{1}{2} \leftrightarrow J=\frac{3}{2}$. Ces résultats seront exposés plus en détail dans une prochaine publication [6].

a) Le rapport ${ }_{\beta} \rho_{0}^{0}(0) !_{\beta} \rho_{2}^{0}(0)$ est indépendant de l'intensité d'irradiation $i_{\lambda}$, aussi bien pour les niveaux $b$ et $a$ de la transition la serque pour les niveaux $c$ atteints par cascade [5] [7].

Avec les notations introduites dans l'article (I), nous avons les relations suivantes

$$
\begin{aligned}
& \frac{{ }_{b} \rho_{0}^{2}(0)}{{ }_{b} \rho_{0}^{0}(0)}= \\
& =(-)^{J_{a}+J_{b}+1} \sqrt{\frac{3\left(2 J_{b}+1\right)}{2}} \cdot\left\{\begin{array}{ccc}
1 & 1 & 2 \\
J_{b} & J_{b} & J_{a}
\end{array}\right\} \cdot \frac{\Gamma_{b}(0)}{\Gamma_{b}(2)} \\
& \frac{{ }_{a} \rho_{0}^{2}(0)}{{ }_{a} \rho_{0}^{0}(0)}= \\
& =(-)^{J_{a}+J_{b}+1} \sqrt{\frac{3\left(2 J_{a}+1\right)}{2}} \cdot\left\{\begin{array}{ccc}
1 & 1 & 2 \\
J_{a} & J_{a} & J_{b}
\end{array}\right\} \cdot\left(\frac{\Gamma_{a}(0)}{\Gamma_{a}(2)}\right)^{*}
\end{aligned}
$$

où

$$
\left(\frac{\Gamma_{a}(0)}{\Gamma_{a}(2)}\right)^{*}=\frac{\Gamma_{a}(0)}{\Gamma_{a}(2)} \cdot \frac{1-\mathcal{A}_{b a}^{(2)} \cdot \frac{\gamma_{b a}}{\Gamma_{b}(2)}}{1-\frac{\gamma_{b a}}{\Gamma_{b}(0)}}
$$

A l'aide de la relation (I, 24), nous obtenons

$$
{ }_{c} \frac{\rho_{0}^{2}(0)}{\rho_{0}^{0}(0)}=\frac{\Theta(b, c, 2)}{\Theta(b, c, 0)} \cdot \frac{\Gamma_{c}(0)}{\Gamma_{c}(2)} \cdot \frac{{ }_{b} \rho_{0}^{2}(0)}{{ }_{b} \rho_{0}^{0}(0)}
$$


qui peut encore s'écrire :

$$
\begin{aligned}
\frac{{ }_{c} \rho_{0}^{2}(0)}{{ }_{c} \rho_{0}^{0}(0)}=( & -)^{J_{a}+2 J_{b}+J_{c}}\left(2 J_{b}+1\right) \sqrt{\frac{3\left(2 J_{c}+1\right)}{2}} \times \\
& \times\left\{\begin{array}{ccc}
1 & 1 & 2 \\
J_{b} & J_{b} & J_{a}
\end{array}\right\} \cdot\left\{\begin{array}{lll}
J_{b} & J_{b} & 2 \\
J_{c} & J_{c} & 1
\end{array}\right\} \times \\
& \times \frac{\Gamma_{b}(0) \cdot \Gamma_{c}(0)}{\Gamma_{b}(2) \cdot \Gamma_{c}(2)} .
\end{aligned}
$$

L'invariance de $R_{\beta \alpha}$ par rapport à $i_{\lambda}$ a déjà été vérifiée pour le niveau supérieur des transitions $1 \rightarrow 0$ et $1 \rightarrow 1$ [3] [8]. Nous présenterons plus loin les vérifications obtenues pour le niveau inférieur, et les niveaux atteints par cascade $\left({ }^{4}\right)$.

b) $\mathrm{Au}$ deuxième ordre en fonction du champ électrique de l'onde laser, l'alignement transversal ${ }_{\beta} \rho_{ \pm 2} 2$ présente, en fonction du champ magnétique une variation lorentzienne [4] :

$$
{ }_{\beta} \rho_{ \pm 2}^{2}=K \cdot \frac{1}{\Gamma_{\beta}(2) \mp 2 i g_{\beta} \mu_{\beta} H} .
$$

Pour le niveau inférieur de la transition, cela suppose évidemment que l'on a séparé l'effet d'excitation directe de l'effet de cascade. On peut montrer [6] que si l'on tient compte des ordres supérieurs en fonction du champ électrique de l'onde laser, pour des intensités pas trop fortes, ${ }_{\beta} \rho_{ \pm 2}^{2}$ a encore une variation lorentzienne, mais que le taux de relaxation $\Gamma_{\beta}(2)$ est remplacé par un taux $\Gamma_{\beta}^{\left(i_{\lambda}\right)}(2)$ dépendant de l'intensité laser.

$$
\Gamma_{\beta}^{\left(i_{\lambda}\right)}(2)=\Gamma_{\beta}(2)+\Delta^{\left(i_{\lambda}\right)} \Gamma_{b}(2)
$$

où $\Delta^{\left(i_{\lambda}\right)} \Gamma_{\beta}(2)$ est proportionnel à l'intensité $i_{\lambda}$, la constante de proportionnalité étant indépendante des caractéristiques du milieu atomique (pression, intensité de décharge, gaz perturbateur). Cette propriété provient du fait qu'il n'y a pas de transfert induit par le laser entre les alignements des niveaux supérieur et inférieur de la transition. (Rappelons que ceci n'est valable que pour les transitions $1 \leftrightarrow 0,1 \leftrightarrow 1$ et $\frac{1}{2} \leftrightarrow \frac{3}{2}$.) Dans ce cas, l'effet Hanle de transfert, obtenu sur les niveaux $c$ atteints par cascade, variera encore comme $\mathfrak{C}_{\alpha}(x)$, mais avec un coefficient $\alpha$ dépendant de l'intensité $i_{\lambda}$ : (équations $1,21,22$ et 24 )

$$
\alpha=\frac{\Gamma_{c}(2)}{\Gamma_{b}^{\left(i_{\lambda}\right)}(2)} \cdot \frac{g_{b}}{g_{c}} .
$$

B. Le niveau $2 p_{4}$; irradiation laser $2 s_{2}-2 \quad p_{1}$ $(\lambda=\mathbf{1 , 5 2} \mu)$. La raie laser utilisée pour l'excitation, $\lambda=1,52 \mu$, est résonnante pour la transition $2 s_{2}-2 p_{1}\left(J_{b}=1 \rightarrow J_{a}=0\right)$.

(4) Pour une transition laser entre niveaux de moments cinétiques quelconques, les relations données dans ce paragraphe sont encore valables lorsqu'on fait tendre l'intensité d'irradiation vers 0 [4].
1. Forme DE LA COURBE DE DÉPOlARISATION MAGNÉTIQUE. - Avec la méthode d'opposition précédemment décrite, nous pouvons observer simultanément l'effet Hanle du niveau $2 s_{2}$, sur la raie infra-rouge $2 s_{2}-2 p_{10}(\lambda=8868 \AA)$ et l'effet Hanle de transfert du niveau $2 p_{4}$ sur les raies visibles $2 p_{4}-1 s_{n}$ (Fig. 3). Nous avons vérifié que la forme de toutes les courbes obtenues pour le niveau $2 s_{2}$ était une forme de Lorentz, $\left[(\Delta H)_{2 s_{2}}^{2}+H^{2}\right]^{-1}$. Ceci nous a permis d'avoir, avec une précision relativement bonne, la largeur $(\Delta H)_{2 s_{2}}$ et d'en déduire le taux de relaxation de l'alignement $\Gamma_{2 s_{2}}^{i_{\lambda}}(2)$. Pour des faibles intensités d'irradiation, $\Gamma_{2 s_{2}}^{i_{\lambda}}(2)$ varie linéairement avec la puissance $d u$ laser.

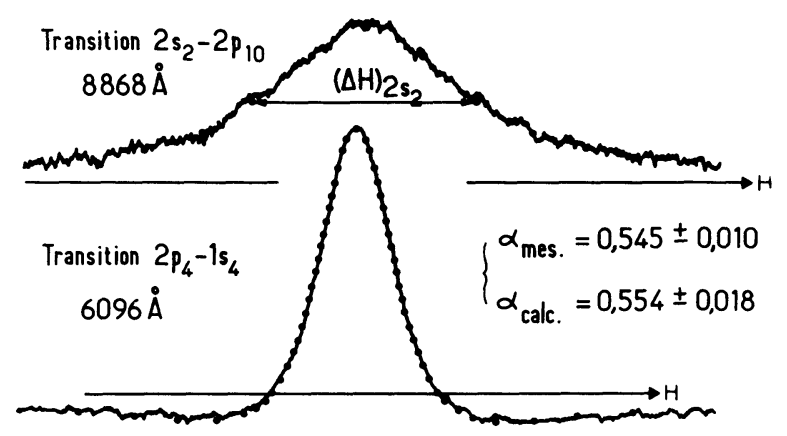

FIG. 3. - Effets Hanle des niveaux $2 s_{2}$ et $2 p_{4}$; irradiation laser $2 s_{2}-2 p_{1}$. Sur cet enregistrement, on a porté l'asymptote choisie et les points théoriques déduits de la valeur de $\alpha$ retenue, $\alpha_{\text {mes. }}=0,545 \pm 0,010$. Cette valeur est en bon accord avec la valeur calculée à partir de résultats antérieurs [2],

$$
\alpha_{\text {calc. }}=0,554 \pm 0,018 \text {. }
$$

La largeur $(\Delta H)_{2 s_{2}}$ ainsi déterminée sert alors "d'unité » pour l'enregistrement considéré et permet de normaliser l'effet Hanle de transfert : il ne reste plus alors qu'à déterminer la valeur du paramètre

$$
\alpha=(\Delta H)_{2 p_{4}} /(\Delta H)_{2 s_{2}}
$$

pour pouvoir comparer l'enregistrement avec la forme de courbe théorique (voir équations I, 19 à I, 22 et II, 13) :

$$
y=\mathcal{C}_{\alpha}(x) \quad \text { où } \quad x=\frac{H}{(\Delta H)_{2 s_{2}}} .
$$

Une détermination grossière de l'asymptote nous permet d'obtenir une première valeur de $\alpha$, par la mesure de la largeur à mi-hauteur. Grâce à cette valeur, il est possible de positionner l'asymptote de façon plus précise par rapport au maximum et aux minima de la courbe. Nous pouvons alors mesurer les largeurs $x(n)$ de la courbe pour des hauteurs $y=n / 20$, $n$ variant de 0 à 19 . (La distance du maximum à l'asymptote est prise égale à 1.) Ces valeurs de $x$, grâce à un calcul-machine établi auparavant [5], permettent de déduire vingt déterminations $\alpha(n)$ de $\alpha$. Si ces valeurs de $\alpha$ concordent, à des fluctuations indépendantes près, l'asymptote est bien placée. Sinon il faut choisir une nouvelle asymptote et recommencer les opérations. 
Nous avons toujours trouvé une position de l'asymptote telle que $\alpha$ soit indépendant de la hauteur, les fluctuations étant inférieures à $3 \%$ (Fig. 4). Ceci prouve que la forme de courbe est correcte : la valeur de $\alpha$ a donc une signification et on peut en déduire la largeur $(\Delta H)_{2 p_{4}}$ et par suite le taux de relaxation de l'alignement $\Gamma_{2 p_{4}}(2)$. Cette grandeur avait déjà été mesurée par une méthode différente, à l'aide de la transition laser $3 s_{2}-2 p_{4}(\lambda=6328 \AA)$ [2]. L'accord entre les deux séries de mesures a pu être vérifié pour différentes valeurs de la pression et de l'intensité de la décharge dans la cellule.

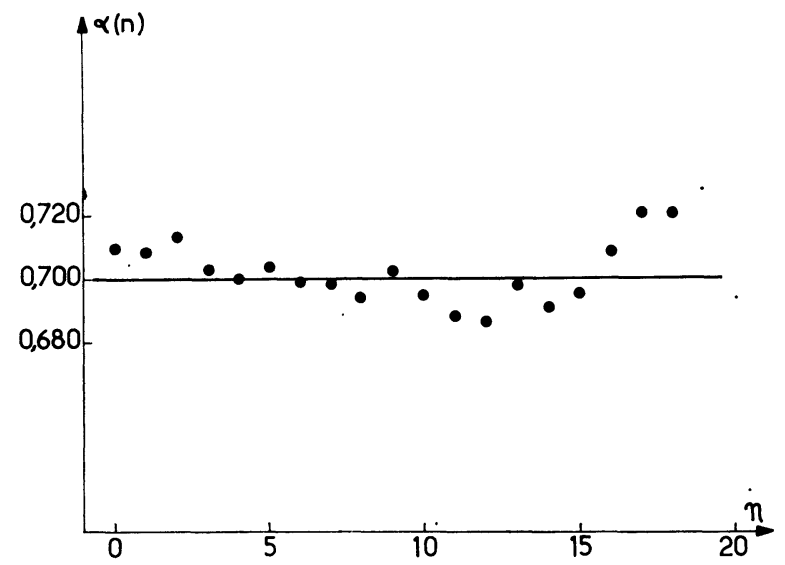

FIG. 4. - Valeurs de $\alpha(n)$ mesurées en fonction de la hauteur $y=n / 20$.

Nous avons vu que l'intensité d'irradiation laser fait varier de manière importante la largeur de l'effet Hanle du niveau $2 s_{2}$. La raie laser n'étant pas en interaction résonnante avec le niveau $2 p_{4}$ ne doit avoir aucune influence sur ce niveau : en agissant sur la puissance du laser, nous avons constaté que la valeur de $\Gamma_{2 p_{4}}(2)$ obtenue restait constante alors que la variation de $\Gamma_{2 s_{2}}^{i_{2}}(2)$ pouvait atteindre $20 \%$ (Fig. 5).

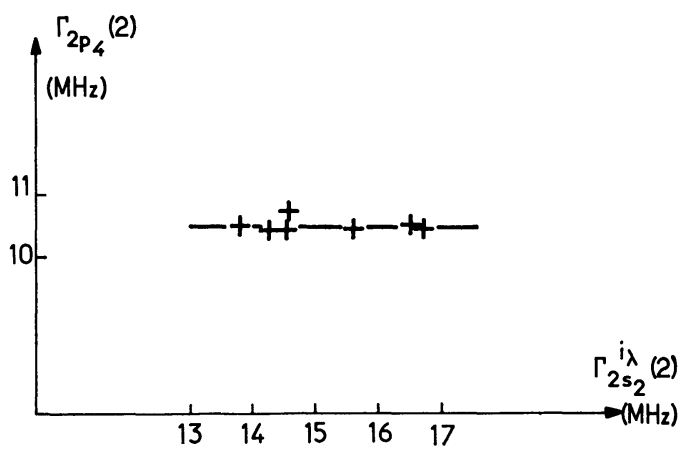

FIG. 5. - Comparaison des variations des taux de relaxation de l'alignement des niveaux $2 s_{2}$ et $2 p_{4}$ sous l'effet de l'intensité laser $i_{\lambda}$ (irradiation laser $2 s_{2}-2 p_{1}$ ).

2. TAUX DE TRANSFERT. - L'observation simultanée, dans les directions $\varphi=0$ et $\varphi=\pi / 2$ (voir Fig. 2) des raies de fluorescence des niveaux $2 s_{2}$ et $2 p_{4}$ et de leurs variations en fonction du champ magnétique (enregistrements reproduits sur la figure $6 a$ et la figure $6 b$ ) nous permet d'atteindre le taux d'aniso-
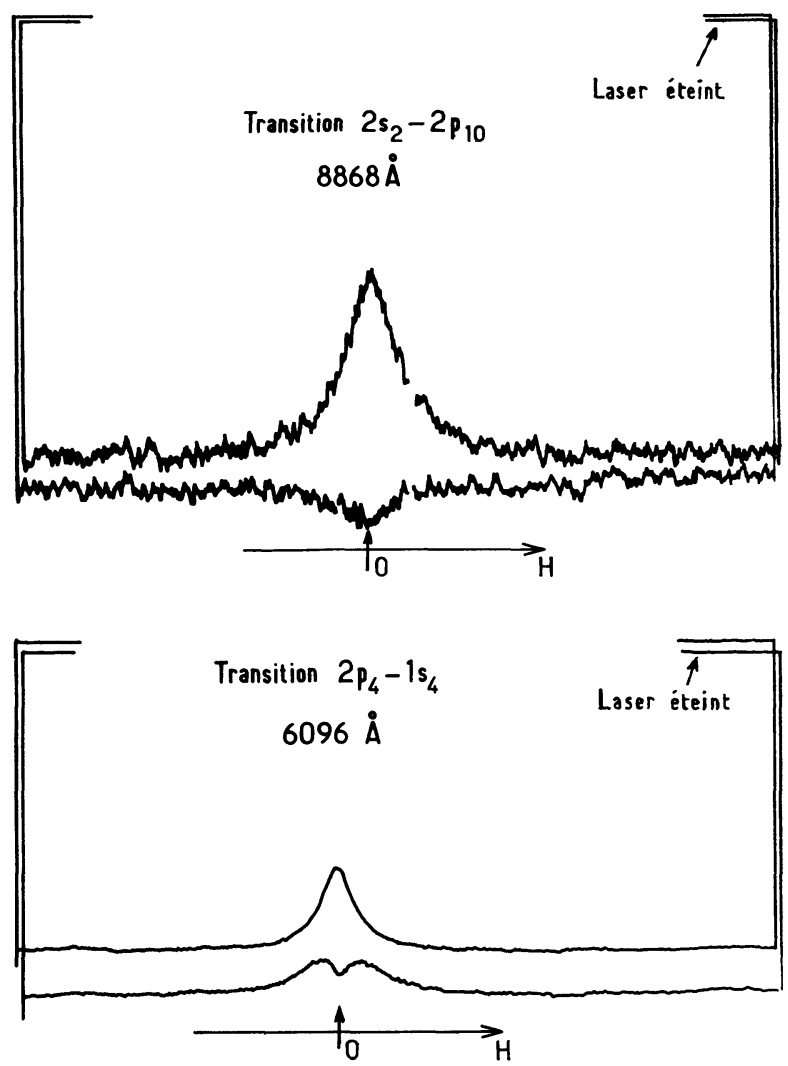

FIG. $6 a$ et $6 b .-$ Variations en fonction du champ magnétique dans les directions $\varphi=0$ et $\varphi=\pi / 2$ (voir Fig. 2), de l'intensité en polarisation $\sigma$ des raies de fluorescence des niveaux $2 s_{2}$ et $2 p_{4}$ (irradiation laser $\left.2 s_{2}-2 p_{1}\right)$. La dissymétrie des signaux observés dans les deux directions est due à la présence, en plus de l'effet Hanle anisotrope (de signe différent dans les deux voies d'observation) d'un terme de saturation isotrope (de même signe dans les deux voies) [4].

tropie en champ magnétique nul, $R$, défini par les équations (II, 3) et (II, 5). Comme la transition $2 s_{2}-2 p_{1}$ est une transition 1-0, le taux d'anisotropie est indépendant de l'intensité laser aussi bien pour le niveau $2 s_{2}\left(R_{2 s_{2}-2 p_{10}}\right)$ que pour le niveau $2 p_{4}\left(R_{2 p_{4}-1 s_{n}}\right)$, ce qui a été vérifié expérimentalement (Fig. 7). La valeur de $R_{2 s_{2}-2 p_{10}}$ nous permet alors d'obtenir, grâce aux relations (II, 5) et (II, 6), $\Gamma_{2 s_{2}}(2) / \Gamma_{2 s_{2}}(0)$. Ensuite, grâce aux relations (II, 5) et (II, 10), $R_{2 p_{4}-1 s_{n}}$ permet d'obtenir $\Gamma_{2 p_{4}}(2) / \Gamma_{2 p_{4}}(0)$.

Nous avons vérifié que nous obtenons toujours la même valeur de $\Gamma_{2 p_{4}}(2) / \Gamma_{2 p_{4}}(0)$ quelle que soit la transition $2 p_{4}-1 s_{n}$ utilisée.

Nous pouvons étudier les variations de

$$
\Gamma_{2 p_{4}}(2) / \Gamma_{2 p_{4}}(0)
$$

en fonction de l'intensité de la décharge $i_{\mathrm{c}}$ et de la pression $p$ de néon, et comparer les valeurs trouvées avec celles obtenues grâce à une irradiation laser à $6328 \AA$ [2]. La figure 8 nous montre la variation du rapport en fonction de $i_{\mathrm{c}}$ à la pression de 1 torr.

La courbe tracée est une courbe calculée à partir des résultats de la référence [2]; on voit que l'accord est excellent. 


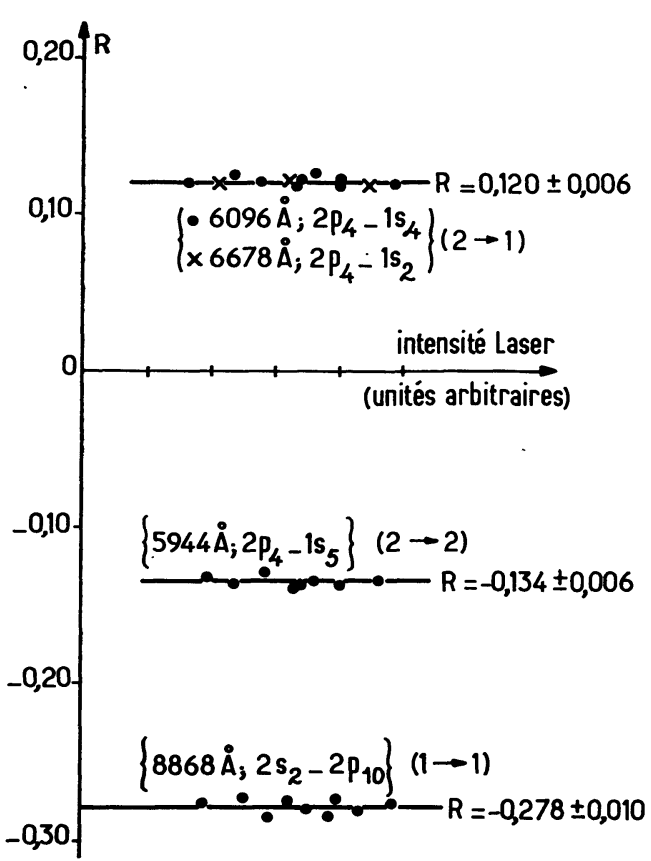

FIG. 7. - Taux d'anisotropie en champ magnétique nul des raies de fluorescence des niveaux $2 s_{2}$ et $2 p_{4}$; irradiation laser $2 s_{2}-2 p_{1}(1 \rightarrow 0) ; \mathrm{Ne} 100 \%, p=1$ torr, $i_{\mathrm{c}}=3 \mathrm{~mA}$. Les valeurs correspondantes de $\Gamma(2) / \Gamma(0)$ sont :

et

$$
\Gamma_{2 s_{2}}(2) / \Gamma_{2 s_{2}}(0)=2,95 \pm 0,10
$$

$$
\Gamma_{2 p_{4}}(2) / \Gamma_{2 p_{4}}(0)=1,41 \pm 0,06 \text {. }
$$

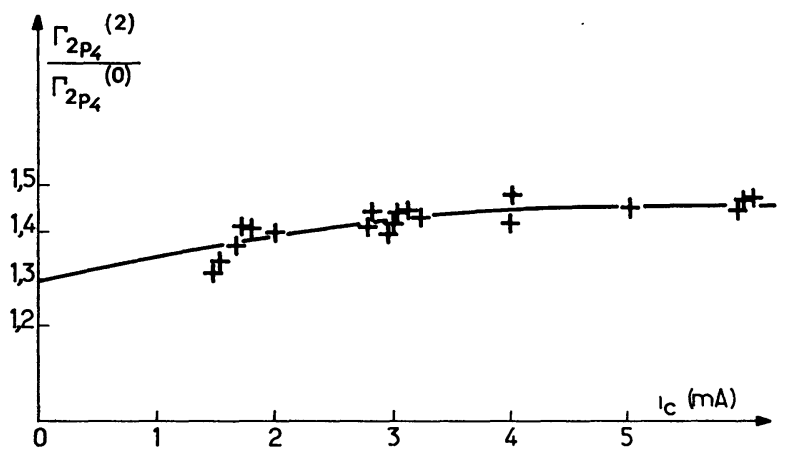

FIG. 8. - Rapport des taux de relaxation de l'alignement et de la population du niveau $2 p_{4}$ en fonction de l'intensité de la décharge; Ne $100 \%, p=1$ torr. La courbe ést tracée à partir des résultats de la référence [2].

Les vérifications expérimentales effectuées sur le $2 p_{4}$ nous ont donc montré que l'étude du transfert (effet Hanle de transfert et taux du transfert d'anisotropie) nous permettait effectivement d'obtenir les temps de relaxation de la population et de l'alignement d'un niveau atteint par cascade.

C. Le niveau $2 p_{2}$; irradiation laser $2 s_{2}-2 p_{1}$ $(\lambda=1,52 \mu)$. - Par la méthode décrite dans le paragraphe précédent, nous étudions la relaxation de la population $\left[\Gamma_{2 p_{2}}(0)\right]$ et de l'alignement $\left[\Gamma_{2 p_{2}}(2)\right] \mathrm{du}$ niveau $2 p_{2}$, en fonction de la pression $p$ et de l'intensité de la décharge $i_{\mathrm{c}}$, dans le néon pur. Nous avons fait varier l'intensité du courant de décharge à pres- sion fixe. Les variations obtenues sont représentées sur la figure 9. A basse pression ( $p<1,5$ torr), $\Gamma(2)$ et $\Gamma(0)$ sont des fonctions décroissantes du courant $i_{\mathrm{c}}$ et tendent vers des valeurs asymptotiques à fort courant, $\Gamma(2)_{\text {as. }}$ et $\Gamma(0)_{\text {as. }}$. A pression plus forte, la variation avec le courant disparaît : ces valeurs indépendantes du courant sont représentées sur la figure 10 en fonction de la pression; on obtient les droites $d_{1}$ et $\mathrm{d}_{2}$. Les valeurs asymptotiques obtenues à faible pression se placent également sur ces deux droites. Ceci laisse supposer que le comportement des $\Gamma$ est le même quelle que soit la pression, mais qu'à forte pression, la décharge s'éteint avant qu'on puisse atteindre des valeurs de $i_{\mathrm{c}}$ assez faibles pour que $\Gamma$
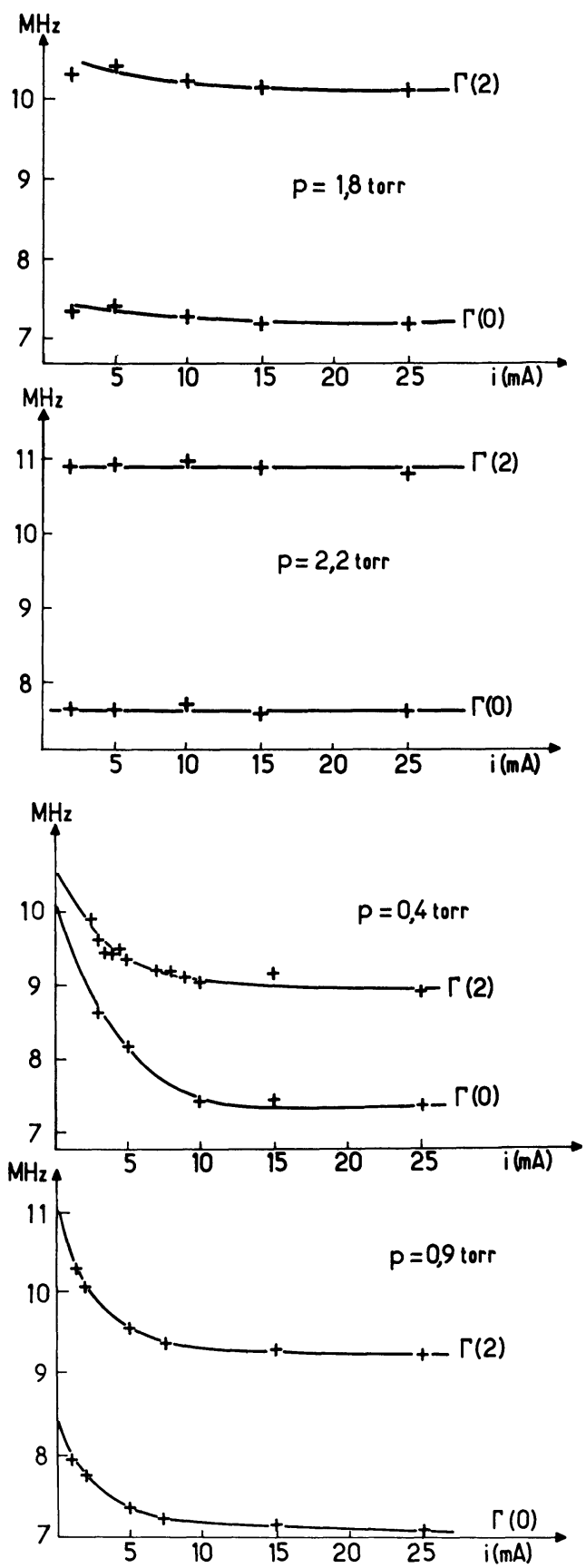

Fig. 9. - Taux de relaxation de l'alignement et de la"population du niveau $2 p_{2}$ en fonction de l'intensité de décharge, pour diverses valeurs de la pression; $\mathrm{Ne} 100 \%$. 


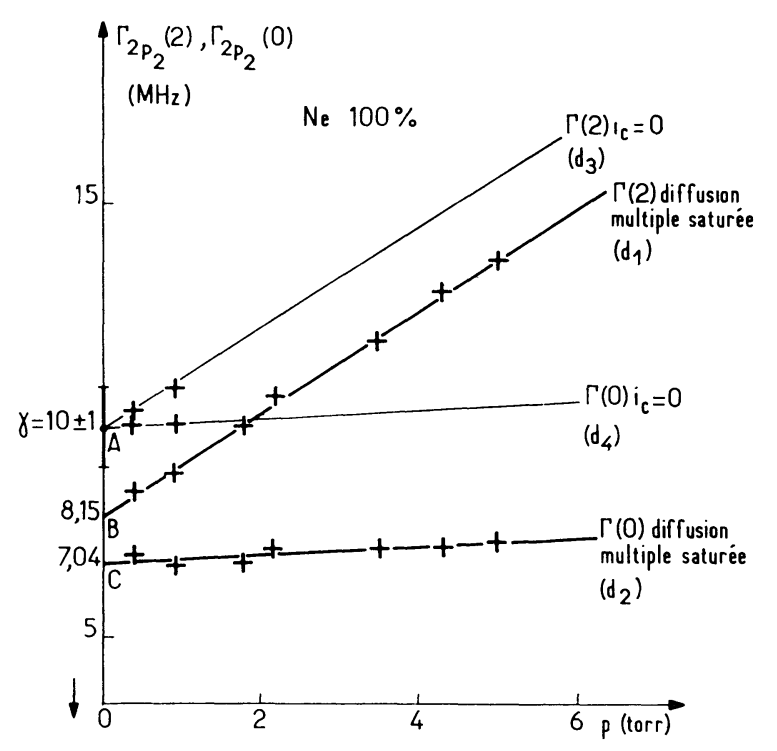

Fig. 10. - Taux de relaxation de l'alignement et de la population du niveau $2 p_{2}$ en fonction de la pression; Ne $100 \%$.

varie. Il n'est donc pas possible, à forte pression, d'extrapoler les $\Gamma$ à $i_{\mathrm{c}}$ nul.

1. DifFUSION MUlTiPle SUR LeS RAIES $2 p_{2}-1 s_{n}$. Nous interprétons ces phénomènes par un effet de diffusion multiple cohérente [9] [10] sur les raies issues du niveau $2 p_{2}$ vers les niveaux $1 s_{n}$. En effet ces niveaux sont très peuplés, car, d'une part les deux niveaux $1 s_{3}(J=0)$ et $1 s_{5}(J=2)$ sont métastables et, d'autre part aux pressions de néon où nous opérons, les raies de résonance des niveaux $1 s_{2}$ et $1 s_{4}$ $(J=1)$ sont entièrement réabsorbées dans la vapeur. La relaxation du niveau $2 p_{2}$ se met alors sous la forme

$$
\Gamma(k)=\gamma\left(1+a_{k} \cdot p-\sum_{n} \alpha_{n}(k) \cdot \lambda_{n} \cdot x_{n}\right)
$$

$\gamma$ est la largeur naturelle du $2 p_{2}, x_{n}$ est le taux de réabsorption [10] de la raie $2 p_{2}-1 s_{n}, \lambda_{n} \cdot \gamma$ la probabilité d'émission spontanée sur cette raie $\left(\sum_{n} \lambda_{n}=1\right)$; $\alpha_{n}(k)$ est un coefficient géométrique [11] qui vaut 1 pour la population $(k=0)$. Pour l'alignement $(k=2)$, $\alpha_{n}(2)$ est égal à $7 / 10$ pour une raie 1-0 $\left(2 p_{2}-1 s_{3}\right)$, $7 / 40$ pour une raie $1-1\left(2 p_{2}-1 s_{2}\right.$ et $\left.2 p_{2}-1 s_{4}\right)$ et $7 / 1000$ pour une raie 1-2 $\left(2 p_{2}-1 s_{5}\right)$. Le terme $a_{k} . p$ représente l'effet des collisions atomiques sur la relaxation de la grandeur tensorielle d'ordre $k$. L'équation (II, 15) montre que les variations de $\Gamma(k)$ en fonction du courant de décharge $i_{\mathrm{c}}$ sont dues uniquement aux variations des taux de réabsorption $x_{n}\left({ }^{5}\right)$. En effet $x_{n}$ est fonction de la densité d'atomes dans le niveau $1 s_{n}$ [8], qui est elle-même une fonction du courant de décharge. Les résultats décrits plus haut indiquent donc que, à fort courant de décharge, $x_{n}$ tend vers une valeur limite. Deux hypothèses sont possibles : soit la den-

(5) L'effet des collisions électroniques et les effets thermiques de la décharge sont supposés négligeables. sité de populations des niveaux $1 s$ devient assez importante, lorsque $i_{\mathrm{c}}$ augmente, pour saturer la diffusion multiple $\left(x_{n} \rightarrow 1\right)$; soit c'est cette densité ellemême qui se sature à fort courant, si bien que $x_{n}$ tend vers une valeur limite inférieure à 1 . Ces deux interprétations vont être successivement envisagées.

$1^{\text {re }}$ hypothèse. - Nous pouvons d'abord supposer que la réabsorption se fait uniquement sur les deux niveaux métastables $1 s_{3}$ et $1 s_{5}$ [7] [12] (la diffusion multiple ne peut pas être saturée sur les quatre niveaux $1 s$, car $\Gamma(0)_{\text {as. }}$ ne tend pas vers zéro en même temps que $p$ ). A faible pression, les taux de réabsorption $x_{3}$ et $x_{5}$ varient de 0 à 1 , quand $i_{\mathrm{c}}$ augmente, et entraînent les variations observées de $\Gamma(2)$ et $\Gamma(0)$. A plus forte pression, la diffusion multiple est saturée quel que soit le courant $\left(x_{3}=x_{5}=1\right)$. Nous pouvons alors en déduire les rapports de branchement $\lambda_{3}$ et $\lambda_{5}$ :

$$
\lambda_{3}=0,27 \pm 0,12 \text { et } \lambda_{5}=0,03 \pm 0,06 .
$$

$\mathrm{La}$ valeur de $\lambda_{3}$ est identique à celle qu'on peut trouver dans les références [13] et [14] mais celle de $\lambda_{5}$ est nettement trop faible. C'est pour cette raison que nous envisagerons une seconde interprétation.

$2^{\mathrm{e}}$ hypothèse. - Des mesures faites sur la densité de populations des niveaux $1 s_{n}$ dans une décharge [15] [16] [17], il ressort que les niveaux $1 s_{2}$ et $1 s_{4}$ sont pratiquement aussi peuplés que les niveaux $1 s_{3}$ et $1 s_{5}$. La densité d'atomes dans ces niveaux augmente avec $i_{\mathrm{c}}$, aux faibles pressions, et tend vers une valeur limite. A plus forte pression, cette densité a une valeur pratiquement indépendante de $i_{\mathrm{c}}$. Ces mesures indiqueraient donc que tous les taux de réabsorption $x_{n}$ varient avec $i_{\mathrm{c}}$ et tendent, quand $i_{\mathrm{c}}$ augmente, vers des valeurs limites inférieures à 1 . Pour que ces valeurs limites soient indépendantes de la pression, il faudrait que les densités correspondantes de métastables le soient aussi. Jusque maintenant, les mesures effectuées l'ont été dans des conditions expérimentales différentes des nôtres et ne nous permettent pas de conclure de façon sûre.

Par ailleurs, nos mesures ont été faites sur un tube à décharge cylindrique de longueur $5 \mathrm{~cm}$ et de diamètre $3 \mathrm{~mm}$. La densité de métastables décroît vite lorsqu'on s'approche des parois du tube [16]. Il est donc possible que la réabsorption des raies soit anisotrope et s'effectue principalement dans l'axe du tube. Si la diffusion multiple n'est pas complètement saturée $\left(x_{n} \neq 1\right)$, le taux de réabsorption sera plus important dans l'axe du tube que dans les directions perpendiculaires. La diffusion aura non plus la symétrie sphérique, mais la symétrie cylindrique autour de $O z$ : dans ces conditions les grandeurs tensorielles $\rho_{q}^{k}$ de même $q$ et de $k$ différents seront couplées entre elles [21]. Dans le cas du $2 p_{2}(J=1)$, l'alignement transversal $\rho_{ \pm 2}^{2}$ restera indépendant des autres observables, mais la population $\rho_{0}^{0}$ et l'alignement longitudinal $\rho_{0}^{2}$ auront des évolutions couplées. Cette anisotropie de la relaxation aura donc peu d'influence sur l'effet Hanle et sur la 
mesure de $\Gamma(2)$, mais pourra changer considérablement le taux d'anisotropie $R$ : la mesure de $\Gamma(0)$ perdra alors toute signification.

2. Collisions $\mathrm{Ne}^{*}-\mathrm{Ne}$. - $\mathrm{Si}$ on néglige les variations possibles des taux de réabsorption limite $x_{n}$ en fonction de la pression, les variations de $\Gamma(2)_{\text {as }}$. et $\Gamma(0)_{\text {as. }}$ en fonction de $p$ (Fig. 10, droites $\mathrm{d}_{1}$ et $\mathrm{d}_{2}$ ) ne sont dues qu'aux seuls termes de collisions atomiques $a_{2} p$ et $a_{0} p$.

On en déduit

et

$$
a_{2}=0,116 \pm 0,005 \text { torr }^{-1} .
$$

$$
a_{0}=0,011 \pm 0,005 \text { torr }^{-1} \text {. }
$$

Etant donné les hypothèses faites (pas de collisions électroniques, pas d'effet thermique de la décharge, invariance par rapport à $p$ du taux limite $x_{n}$, diffusion multiple isotrope), on ne peut accorder qu'une signification qualitative à la valeur de $a_{0}$ : les collisions de « quenching " sont très faibles.

Par contre, d'après ce qu'on a vu dans le paragraphe précédent, on peut accorder une signification quantitative à la valeur importante de $a_{2}$. On peut alors calculer la section efficace de destruction de l'alignement $\left({ }^{6}\right)$ :

$$
\sum_{\mathrm{Ne}^{*-\mathrm{Ne}}}^{(2)}=(31 \pm 4) \times 10^{-16} \mathrm{~cm}^{2}
$$

Cette valeur est en excellent accord avec la valeur théorique $32 \times 10^{-16} \mathrm{~cm}^{2}$, calculée par l'un des auteurs dans le couplage $j l$ de Racah [19], modifié de façon à tenir compte des mélanges de configuration [8].

A faible pression ( $p<1$ torr), il est possible d'extrapoler $\Gamma(2)$ et $\Gamma(0)$ à intensité de décharge nulle (Fig. 9), ce qui réduit la densité de métastables $1 s_{n}$ et élimine la diffusion multiple $\left(x_{n}=0\right)$. Nous pouvons en effet vérifier que les droites $d_{3}$ et $d_{4}$ (Fig 10) passant par les points ainsi obtenus et parallèles à $d_{1}$ et $\mathrm{d}_{2}$ se coupent approximativement à pression nulle pour donner la largeur naturelle $\gamma$ du niveau $2 p_{2}$. Si la diffusion multiple n'était pas éliminée, ces droites ne se couperaient pas à pression nulle. Etant donné la faible précision de cette méthode, $\gamma$ ne peut être obtenu à mieux de $10 \% \gamma=10 \pm 1 \mathrm{MHz}$.

Pour effectuer des mesures plus nettement interprétables et donc plus précises, il faudrait pouvoir éliminer la diffusion multiple quelle que soit la pression du gaz. Ceci est possible dans des mélanges d'hélium

(6) La marge d'erreur pour $\sum_{\mathrm{Ne}^{*}-\mathrm{Ne}}^{(2)}$ est plus importante que celle pour $a_{2}$, car elle tient compte de l'incertitude sur la température de la décharge (qu'on a prise égale à $350^{\circ} \mathrm{K}$ ) et des variations possibles des taux limites $x_{n}$ en fonction de la pression (on peut raisonnablement supposer que ces variations sont inférieures à $a_{0} p$, étant donné que les collisions de quenching sont faibles). et de néon. Malheureusement, les expériences précédentes n'ont pu être effectuées que sur le néon pur. En effet, il existe dans le spectre de l'hélium une raie très intense de longueur d'onde $1,06 \mu$. Cette raie diffuse sur tout le spectre infra-rouge du fait de la qualité très médiocre des réseaux de nos monochromateurs. Il en résulte un bruit de fond très important qui rend trop imprécise toute étude du niveau $2 s_{2}$. Pour contourner cette difficulté, nous avons excité le niveau $2 p_{2}$ par la raie laser $6401 \AA\left(3 s_{2}-2 p_{2}\right)$.

D. Le niveau $2 p_{2}$; irradiation laser $3 \quad s_{2}-2 \quad p_{2}$ $(\lambda=6401 \AA)$. - Dans ce cas, deux effets se superposent : l'excitation directe par le laser et l'excitation par transfert à partir du niveau supérieur $3 s_{2}$ : comme il s'agit d'une raie laser $J_{b}=1 \rightarrow J_{a}=1$, le transfert d'alignement n'est pas négligeable (voir article (I)).

1. TRANSFERT D'ALIGNEMENT SUR LA TRANSITION LASER. - L'importance relative des termes d'excitation directe et de transfert est donnée par (voir équations I, 30 et 34 )

$$
\varepsilon=\frac{\gamma_{b a}}{2 \Gamma_{3 s_{2}}(2)}
$$

où $\gamma_{b a}$ est la probabilité d'émission spontanée sur la transition $3 \quad s_{2}-2 \quad p_{2}$. Des mesures d'intensité de raies [7] nous ont montré que $\gamma_{b a} \lesssim 0,4 \mathrm{MHz}$. Par ailleurs, d'après des résultats obtenus antérieurement [3] [8], $\Gamma_{3 s_{2}}(2)$ est toujours supérieur à $5 \mathrm{MHz}$. Donc $\varepsilon$ est toujours inférieur à $4 \%$. De plus, le coefficient $\alpha^{\prime}$ de l'effet Hanle de transfert (défini par l'équation (I, 30) n'est jamais supérieur à 1 : le transfert d'alignement deviendra négligeable dès que le champ magnétique $\mathrm{H}$ sera supérieur à la largeur de la courbe d'effet Hanle direct (voir Fig. $2 b$ de l'article (I)). Le transfert n'affecte donc que le sommet de la courbe.

La méthode pratique d'analyse de ces courbes consiste à retrouver la forme de Lorentz de l'effet Hanle direct seul, en corrigeant légèrement le sommet. Pour cela, on procède par approximations successives en se servant des ailes de la courbe qui ne sont pas déformées par le transfert. On mesure la largeur $l$ au $1 / 5^{\mathrm{e}}$ de la hauteur de la courbe expérimentale, puis on détermine la hauteur pour laquelle la largeur de la courbe est $l / 2$ : c'est en première approximation la demi-hauteur de la forme de Lorentz recherchée ; on obtient ainsi une nouvelle position du sommet à partir de laquelle on recommence l'opération. La méthode est rapidement convergente, si le rapport signal sur bruit est bon. La figure 11 donne un exemple d'enregistrement sur lequel on a tracé en pointillés la courbe obtenue après élimination du transfert. De la largeur de la courbe ainsi corrigée, on peut déduire le taux de relaxation de l'alignement transversal $\Gamma_{2 p_{2}}^{i_{\lambda}}(2)$. Ce taux dépend de l'intensité laser $i_{\lambda}$ (Fig. 12). La contribution du laser à la relaxation est éliminée par l'extrapolation à intensité nulle : la valeur obtenue, $\Gamma_{2 p_{2}}(2)$ est alors indépendante des caractéristiques du rayon- 


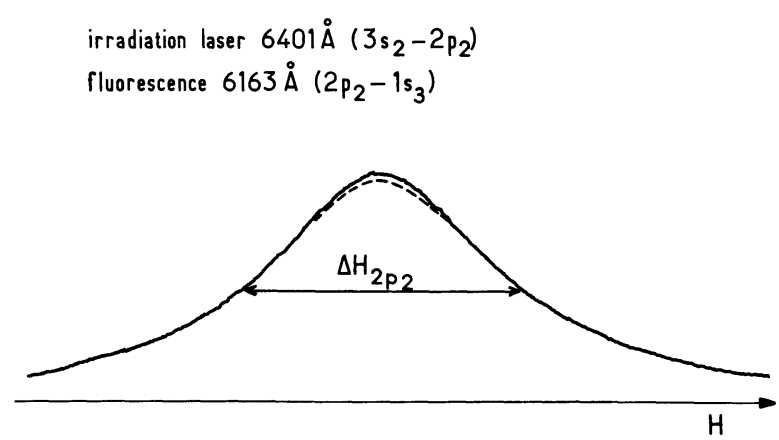

Fig. 11. - Effet Hanle du niveau $2 p_{2}$; on a tracé en pointillés la courbe obtenue après élimination du transfert sur la transition laser $\left(3 s_{2} \rightarrow 2 p_{2}\right)$.

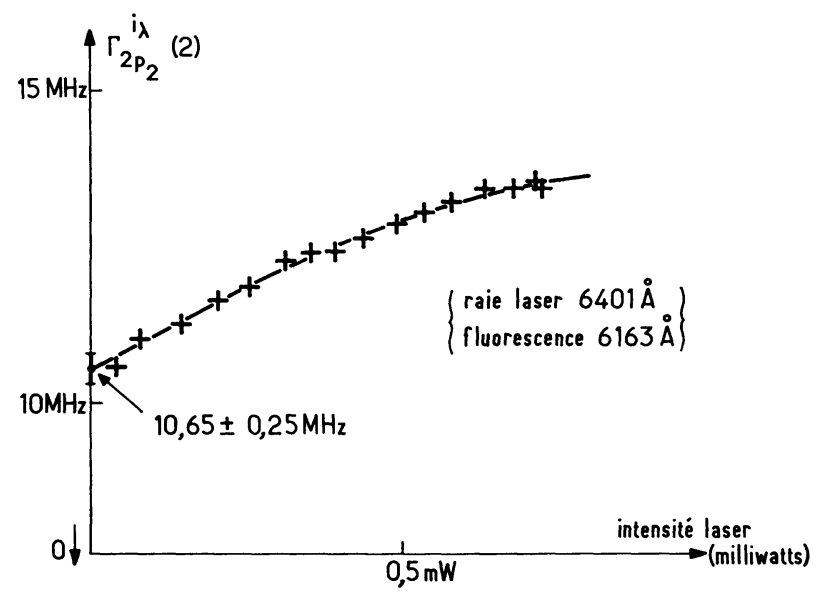

FIG. 12. - Taux de relaxation de l'alignement du niveau $2 p_{2}$ en fonction de l'intensité laser; irradiation $3 s_{2}-2 p_{2}$; Ne $1 \%$ He $99 \%, p=1,10$ torr, $i_{\mathrm{c}}=4,3 \mathrm{~mA}$.

nement. C'est cette valeur qu'il faut comparer avec les mesures faites dans le cas d'une irradiation laser de longueur d'onde $\lambda=1,52 \mu$. L'accord entre les valeurs de $\Gamma(2)$ obtenues par ces deux méthodes dans le néon pur est excellent [7]. Avec l'irradiation laser $\lambda=6401 \AA$, nous pouvons faire des mesures sur des mélanges d'hélium et de néon (ce qui nous permet d'éliminer sans ambiguilté la diffusion multiple) et d'étudier les collisions $\mathrm{Ne}$ - $\mathrm{He}$.

2. Collisions déPolarisantes Ne*-He. - Nous présenterons ici les mesures faites sur un mélange de proportions $1 \%$ néon- $99 \%$ hélium. Dans ce mélange, le nombre d'atomes dans les niveaux métastables $1 \mathrm{~s}$ devient négligeable (tout au moins pour des intensités de décharge et des pressions pas trop fortes) et il n'y a plus diffusion multiple sur les raies correspondantes. A la précision des mesures, $\Gamma_{2 p_{2}}(2)$ est alors indépendant de l'intensité de décharge et sa variation en fonction de la pression est linéaire (Fig. 13)

$$
\Gamma_{2 p_{2}}(2)=\gamma\left(1+b_{2} p\right)
$$

avec

$$
\gamma=9,40 \pm 0,40 \mathrm{MHz} \text { et } b_{2}=0,133 \pm 0,012 \text { torr }^{-1}
$$

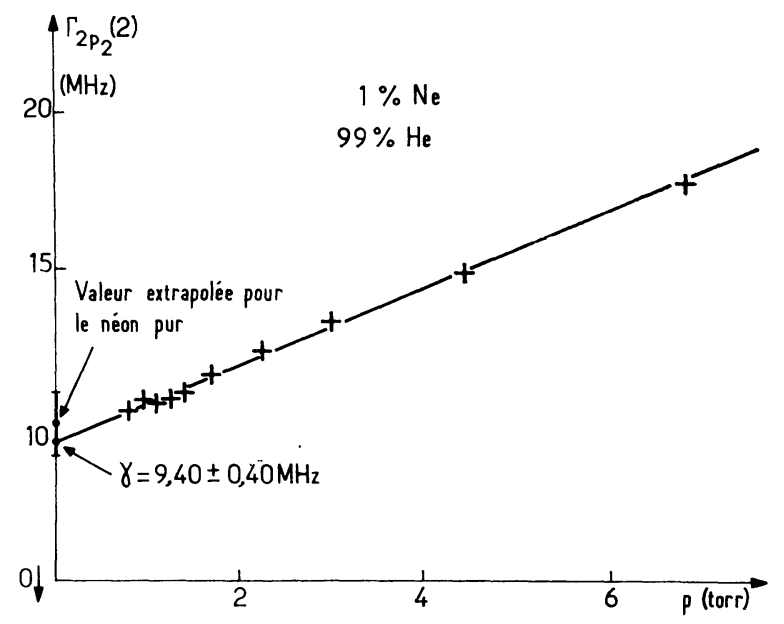

Fig. 13. - Taux de relaxation de l'alignement du niveau $2 p_{2}$ en fonction de la pression; $\mathrm{Ne} 1 \%$, He $99 \%$.

ce qui donne pour la durée de vie naturelle

$$
\tau=(16,9 \pm 0,8) \times 10^{-9} \mathrm{~s}
$$

et pour la section efficace de collision

$$
\sum_{\mathrm{Ne}^{*}-\mathrm{He}}^{(2)}=(13 \pm 2) \times 10^{-16} \mathrm{~cm}^{2} \cdot\left({ }^{7}\right)
$$

Cette dernière valeur est en excellent accord avec la valeur théorique $12,5 \times 10^{-16} \mathrm{~cm}^{2}$ [8].

Par ailleurs, la valeur de $\gamma$ obtenue ici confirme et précise les mesures effectuées sur le néon pur.

3. TRANSFert DE POPUlation ET COLlisions DE QUENCHING Ne*-He. DÉTERMINATION DE LA PROBABILITÉ D’ÉMISSION SPONTANÉE $3 s_{2} \rightarrow 2 p_{2}$. - Nous allons maintenant présenter les résultats obtenus grâce à l'étude du taux d'anisotropie $R$ des raies de fluorescence du niveau $2 p_{2}$. Nous avons vu que pour le niveau inférieur d'une transition laser $1-1$, ce taux doit être indépendant de l'intensité laser $i_{\lambda}$. Ceci a été vérifié expérimentalement (Fig. 14). Les valeurs de $R$ pour les diverses raies de fluorescence concordent pour donner une même valeur de

$X=\left[\frac{\Gamma_{a}(2)}{\Gamma_{a}(0)}\right]_{2 p_{2}}^{*}=\frac{\Gamma_{2 p_{2}}(2)}{\Gamma_{2 p_{2}}(0)} \cdot \frac{1-\gamma_{b a} / \Gamma_{3 s_{2}}(0)}{1+\gamma_{b a} / 2 \Gamma_{3 s_{2}}(2)}$

(voir équations II, 5, 7, 8).

Dans un mélange de proportions $1 \%$ néon-99 \% hélium, $X$ augmente légèrement avec l'intensité de la décharge $i_{\mathrm{c}}$, et la valeur extrapolée à intensité nulle, présente, en fonction de la pression, les variations représentées sur la figure 15 . On peut constater qu'à pression nulle, $X$ tend vers une valeur limite infé-

(7) Nous avons pris ici $T=300^{\circ} \mathrm{K}$, comme température du milieu atomique; en effet, dans ce mélange Hélium-Néon, nous avons pu extrapoler les mesures effectuées à intensité de décharge nulle, ce qui n'était pas le cas pour le Néon pur (voir Note 6). 


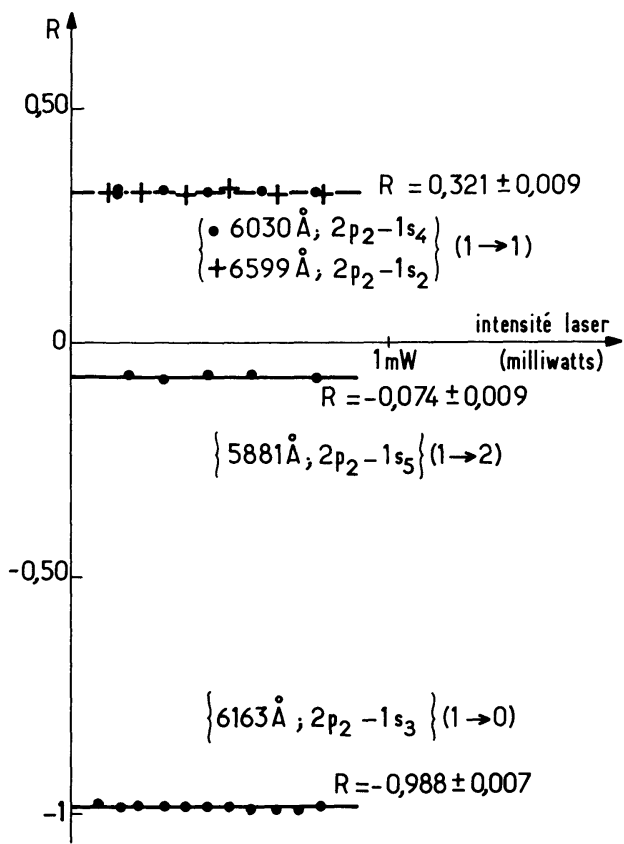

Fig. 14. - Taux d'anisotropie en champ magnétique nul des raies de fluorescence du niveau $2 p_{2}$; irradiation laser $3 s_{2}-2 p_{2}$ $(1 \rightarrow 1) ; \mathrm{Ne} 10 \%$, He $90 \% ; p=0,8$ torr, $i_{\mathrm{c}}=6,3 \mathrm{~mA}$. La valeur correspondante de $\left[\frac{\Gamma_{a}(2)}{\Gamma_{a}(0)}\right]_{2 p_{2}}^{*}$ est

$$
X=1,010 \pm 0,006 \text {. }
$$

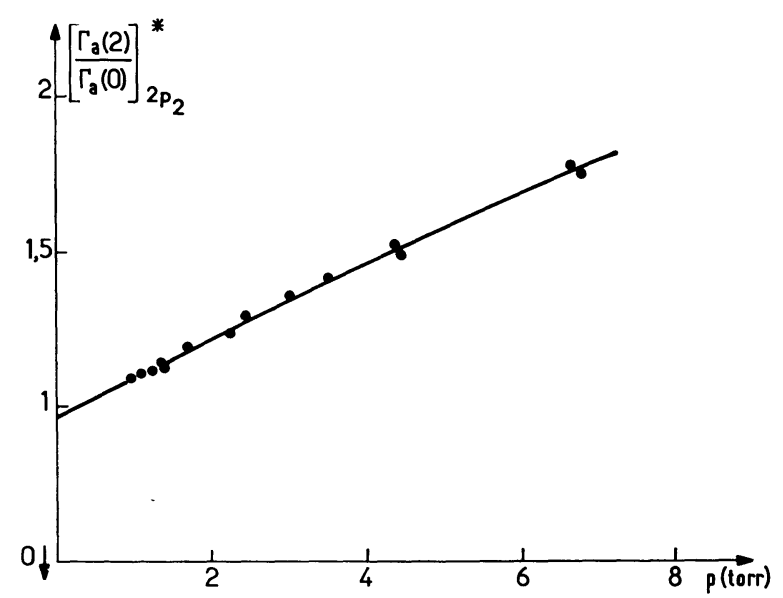

FIG. 15. - Variation de $\left[\frac{\Gamma_{a}(2)}{\Gamma_{a}(0)}\right]_{2 p_{2}}^{*}$ en fonction de la pression ; irradiation laser $2 s_{2}-2 p_{2}$; Ne $1 \%$-He $99 \%$.

rieure à 1 . D'après la formule (II, 18), cette valeur limite doit être égale à

$$
X(p=0)=\frac{1-\gamma_{b a} / \gamma_{3 s 2}}{1+\gamma_{b a} / 2 \gamma_{3 s_{2}}}
$$

En principe, on peut en déduire la probabilité de transition $\gamma_{b a}$ et, par suite, obtenir $\Gamma_{2 p_{2}}(0)$, à partir de la formule (II, 18).

Cependant comme cette méthode est peu précise, nous avons employé une autre méthode qui a été mise au point sur le niveau $2 p_{4}$ [2] [8]. Nous considérons la quantité :

$$
t=\frac{\Gamma_{2 p_{2}}(0)}{\gamma_{2 p_{2}}}=\frac{1}{X} \cdot \frac{\Gamma_{2 p_{2}}(2)}{\gamma_{2 p_{2}}} \cdot \frac{1-\gamma_{b a} / \Gamma_{3 s_{2}}(0)}{1+\gamma_{b a} / 2 \Gamma_{3 s_{2}}(2)} .
$$

$t$ doit avoir une variation linéaire en fonction de la pression et tendre vers 1 quand $p$ tend vers $0 . \Gamma_{2 p_{2}}(2)$ et $\gamma_{2 p_{2}}$ sont déduits des mesures précédentes; $\Gamma_{3 s_{2}}(2)$ et $\Gamma_{3 s_{2}}(0)$ sont connus par des expériences antérieures [3] ; $X$ est mesuré. On se donne alors une série de valeurs d'essai pour $\gamma_{b a}$; pour chacune de ces valeurs, on calcule $t(p)$ et on trace la droite moyenne représentant les variations de $t$ en fonction de $p$. C'est la droite qui passe par la valeur 1 à pression nulle qui donne la valeur de $\gamma_{b a}$.

Nous avons obtenu ainsi $\gamma_{b a}=0,18 \pm 0,09 \mathrm{MHz}$ ou encore $\gamma_{b a}=1,2 \pm 0,5 \times 10^{6} \mathrm{~s}^{-1}$.

A notre connaissance, la probabilité de transition de la raie $\lambda=6401 \AA\left(3 s_{2}-2 p_{2}\right)$ n'avait pas encore été mesurée. Par une méthode analogue, la probabilité de transition de la raie $6328 \AA\left(3 s_{2}-2 p_{4}\right)$ a pu être mesurée [3] :

$$
\gamma_{b a}=0,51 \pm 0,08 \mathrm{MHz} .
$$

Le rapport des deux probabilités est de 0,34 , valeur très comparable avec la valeur théorique 0,40 , calculée dans le couplage $j-l$ de Racah [19]. La droite $t(p)$ correspondant à la valeur de $\gamma_{b a}$ retenue est reproduite sur la figure 16 . On en déduit

$$
\left(\frac{\Gamma_{a}(0)}{\gamma_{a}}\right)_{2 p_{2}}=1+b_{0} p
$$

avec $b_{0}=0,005 \pm 0,004$ torr $^{-1}$.

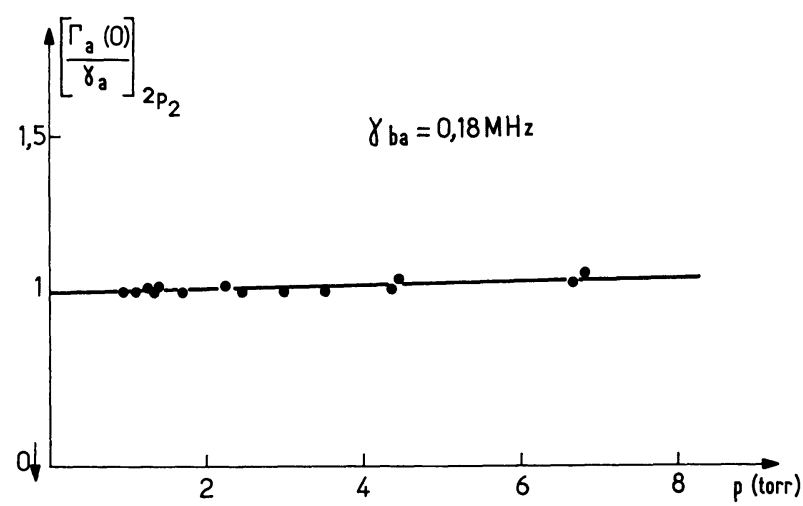

FIg. 16. - Taux de relaxation de la population du niveau $2 p_{2}$ en fonction de la pression; Ne $1 \%$, He $99 \%$.

On notera la faible importance des collisions de quenching qui apparaissent à la limite de la précision expérimentale. $\gamma_{b a}$ connu, on peut étudier les variations de $\Gamma_{2 p_{2}}(0)$ en fonction du courant de décharge ; on trouve, toujours à la limite des erreurs expérimentales, un très faible affinement (de l'ordre de $1 \%$ par $\mathrm{mA}$ et par torr). On peut l'attribuer soit à un début de réabsorption de la raie $2 p_{2}-1 s_{5}$, car le métastable $1 s_{5}$ est encore assez peuplé dans les mélanges d'hélium et de néon [16] [17] soit à un effet thermique de la décharge [2] [8]. Il n'est pas possible de trancher de façon sûre. 
Conclusion. - Des publications antérieures [2] [3] [4] avaient montré que le pompage optique à l'aide d'une raie laser permettait d'étudier les caractéristiques du niveau supérieur de la transition laser, ainsi que celles du niveau inférieur dans le cas où le transfert de cohérence Zeeman par émission spontanée est négligeable. Nous avons montré ici comment étendre cette méthode :

a) aux niveaux atomiques qui ne sont pas en interaction directe avec la raie laser, mais qui sont ali- mentés par cascades radiatives à partir du niveau supérieur de la transition laser ;

b) au niveau inférieur de la transition laser, quand le transfert de cohérence Zeeman sur cette transition n'est plus négligeable.

Nous avons de plus mesuré quelques paramètres nouveaux du niveau $2 p_{2}$. Malheureusement la diffusion multiple sur les métastables rend ces mesures peu précises.

\section{Bibliographie}

[1] Ducloy (M.) et Decomps (B.), C. R. Acad. Sc., 1968, $266 \mathrm{~B}, 412$.

[2] Decomps (B.) et Dumont (M.), J. Physique, 1968, 29, 443.

[3] Decomps (B.) et Dumont (M.), I. E. E. E., J. Quant. Electr., 1968, QE 4, 916.

[4] Dumont (M.) et Decomps (B.), J. Physique, 1968, 29, 181.

[5] Ducloy (M.), Thèse de 3e cycle, Paris, 1968.

[6] Dumont (M.) et Ducloy (M.), à paraître.

[7] Fournier (E.), Thèse de $3^{\mathrm{e}}$ cycle, Paris, 1969.

[8] Decomps (B.), Thèse, Paris, 1969.

[9] Barrat (J. P.), J. Phys. Rad., 1959, 20, 541, 633 et 657.

[10] Omont (A.), J. Physique, 1965, 26, 576.

OMONT (A.), Thèse, Paris, 1967.

[11] Saloman (E. B.) et Happer (W.), Phys. Rev., 1966, 144,7

[12] Fournier (E.), Ducloy (M.), Decomps (B.) et Dumont (M.), C. R. Acad. Sc., 1969, 268 B, 1495.

[13] Phelps (A. V.), Phys. Rev., 1959, 114, 1011.

A partir des valeurs retenues par PhelPs, nous pouvons obtenir : $\lambda_{2}=0,47 ; \lambda_{3}=0,29 ; \lambda_{4}=0,09$; $\lambda_{5}=\mathbf{0}, 15$

[14] Wiese (W. L.), SMith (M. W.) et Glennon (B. M.), Atomic transition probabilities, Vol. I, Hydrogen trough Neon, NSRDS, NBS4, Washington, 1966.
Les valeurs suivantes sont proposées : $\lambda_{2}=0,42$; $\lambda_{3}=0,27 ; \lambda_{4}=0,10 ; \lambda_{5}=0,21$.

[15] Delpech (J. F.), Thèse, Orsay, 1968.

[16] Schlie (L. A.) et Verdeyen (J. T.), I. E. E. E., J. Quant. Electr., 1969, QE 5, 21.

Miller (P. A.), Verdeyen (J. T.) et Cherrington (B. E.), I. E. E. E., J. Quant. Electr., 1969, QE 5, 473.

[17] Ricard (A.), J. Physique, 1969, 30, 556.

[18] Cette valeur de la durée de vie naturelle du $2 p_{2}$ est en bon accord avec celles proposées par KLOSE : $16,3 \pm 0,6 \mathrm{~ns}$ et $16,8 \pm 0,7 \mathrm{~ns}$.

Klose (J. Z.), Phys. Rev., 1966, 141, 181.

Par contre, la valeur obtenue par BENNETT est nettement supérieure : $\tau=18,8 \pm 0,3 \mathrm{~ns}$

Bennett (W. R.) et Kindlmann (P. J.), Phys. Rev., 1966, 149, 38.

Il en est de même pour celle proposée par Oshiro$\mathrm{VICH}: 20 \pm 1,6 \mathrm{~ns}$.

Oshirovich (A. L.) et Verolainen (Y. F.), Optics, and Spectroscopy, 1967, 22, 181.

[19] Racah (G.), Phys. Rev., 1942, 61, 537.

[20] Burla Macchi (P.) et Toraldo di Francia (G.), $I l$ Nuovo Cimento, 1966, 42, BN 1, 1310.

Tobias (I.) et Strouse (W. M.), Appl. Phys. Lett., $1967,10,342$.

[21] Lombardi (M.), C. R. Acad. Sci., 1967, 265, 191. 